\title{
A NEW ANTISYMMETRIC BILINEAR MAP FOR TYPE-I GAUGE THEORIES
}

\author{
Giampiero Esposito, ${ }^{1,2}$ Cosimo Stornaiolo ${ }^{1,2}$ \\ ${ }^{1}$ Istituto Nazionale di Fisica Nucleare, Sezione di Napoli, \\ Complesso Universitario di Monte S. Angelo, \\ Via Cintia, Edificio 6, 80126 Napoli, Italy \\ ${ }^{2}$ Dipartimento di Scienze Fisiche, Complesso Universitario di Monte S. Angelo, \\ Via Cintia, Edificio 6, 80126 Napoli, Italy
}

(Dated: August 11, 2021)

\begin{abstract}
In the case of gauge theories, which are ruled by an infinite-dimensional invariance group, various choices of antisymmetric bilinear maps on field functionals are indeed available. This paper proves first that, within this broad framework, the Peierls map (not yet the bracket) is a member of a larger family. At that stage, restriction to gauge-invariant functionals of the fields, with the associated Ward identities and geometric structure of the space of histories, make it possible to prove that the new map is indeed a Poisson bracket in the simple but relevant case of Maxwell theory. The building blocks are available for gauge theories only: vector fields that leave the action functional invariant; the invertible gauge-field operator, and the Green function of the ghost operator.
\end{abstract}




\section{INTRODUCTION}

In the global approach to quantum field theory [1], the physical arena is given by the infinite-dimensional manifold $\Phi$ of field histories $\varphi^{i}$. For gauge fields, there exists on $\Phi$ a set of vector fields $Q_{\alpha}$ that leave the action $S$ invariant, i.e.

$$
Q_{\alpha} S=0
$$

On denoting by $Q_{\alpha}^{i}$ the components of the $Q_{\alpha}$, one can write Eq. (1.1) in the form

$$
S_{, i} Q_{\alpha}^{i}=0
$$

the comma being hereafter the standard notation for functional derivatives $\frac{\delta}{\delta \varphi^{i}}$ with respect to field variables [1]. The vector fields $Q_{\alpha}$ are linearly independent, and for type-I gauge theories the Lie brackets of the $Q_{\alpha}$ depend linearly on the $Q_{\alpha}$ themselves:

$$
\left[Q_{\alpha}, Q_{\beta}\right]=C_{\alpha \beta}^{\gamma} Q_{\gamma}
$$

where the $C_{\alpha \beta}^{\gamma}$ are independent of field variables: $C_{\alpha \beta, i}^{\gamma}=0$, and are therefore called 'structure constants'. The proper gauge group $\mathcal{G}$ is the set of transformations of $\Phi$ into itself obtained by exponentiating the infinitesimal gauge transformation

$$
\delta \varphi^{i}=Q_{\alpha}^{i} \delta \xi^{\alpha}
$$

and taking products of the resulting exponential maps [1]. The closure property expressed by Eq. (1.3) implies that the proper gauge group decomposes $\Phi$ into subspaces, known as orbits, to which the $Q_{\alpha}$ are tangent. The space of orbits is the quotient space $\Phi / \mathcal{G}$.

In recent work by the authors [2], we have considered the problem of defining the most general Poisson bracket on the space of gauge-invariant functionals for type-I theories. Our original hope was that, in the same way as the Moyal bracket [3, 4, 5] is proportional to the Poisson bracket to lowest order in $\hbar$, one might be able to define a new Poisson bracket that is proportional to the Peierls bracket [6] to lowest order in $\hbar$. In the latter, the building block is the supercommutator function, i.e. the difference

$$
\widetilde{G}^{j k} \equiv G^{+j k}-G^{-j k}=-\widetilde{G}^{k j}
$$

of advanced and retarded Green functions of the invertible operator $F_{i j}$ on gauge fields $\varphi^{j}$ obtained after adding a gauge-breaking term in the functional integral [1]. [By virtue of 
the definition (1.5), the integral representation of $\widetilde{G}^{j k}$ is given by a contour in the complex $p^{0}$-plane passing below the poles on the real line] We were thus looking for a quantum commutator having the following asymptotic expansion:

$$
[A, B] \sim i \hbar(A, B)+\mathrm{O}\left(\hbar^{3}\right)
$$

where $A$ and $B$ are any two gauge-invariant functionals:

$$
Q_{\alpha} A=Q_{\alpha} B=0
$$

and $(A, B)$ is their Peierls bracket [1, 2]

$$
(A, B) \equiv A_{, i} \widetilde{G}^{i j} B_{, j}=\iint d x d y \frac{\delta A}{\delta \varphi^{i}(x)} \widetilde{G}^{i j}(x, y) \frac{\delta B}{\delta \varphi^{j}(y)} .
$$

With hindsight, we were misled in putting the emphasis on Moyal brackets, since the phasespace structure is totally extraneous to a space-of-histories formulation. With this improved understanding, section 2 builds a map, not yet a Poisson bracket, which is antisymmetric and bilinear. Sections 3 and 4 exploit the geometry of the space of histories to give a concrete form to our new map, and show under which conditions it becomes a Poisson bracket. Section 5 derives its gauge transformation properties. Concluding remarks and open problems are presented in section 6 .

\section{AN ANTISYMMETRIC BILINEAR MAP}

Given the space of histories $\Phi$ for type-I theories, with the associated space of functionals $\mathcal{F}(\Phi)$, suppose we want to define a suitable map (not just its asymptotic expansion)

$$
[,]: \mathcal{F}(\Phi) \times \mathcal{F}(\Phi) \rightarrow \mathcal{F}(\Phi)
$$

acting on any two field functionals $A$ and $B$ according to

$$
[A, B] \equiv i \hbar(A, B)+\mu_{3}(i \hbar)^{3} A_{, j k l} U^{j p} U^{k q} U^{l r} B_{, p q r}
$$

Note that, at this stage, $(2.1)$ is not a Poisson bracket, since the Peierls map $(A, B)$ (whose action on $A$ and $B$ is analogous to (1.8)) is not a Poisson bracket for generic $A$ and $B$. The coefficient $\mu_{3}$ of $(i \hbar)^{3}$ should be so chosen that the two terms on the right-hand side of $(2.1)$ 
have the same dimension. However, we stress that the form of $U^{j p}$ is not fixed a priori. From the definition (2.1) we find

$$
[B, A]=-i \hbar(A, B)+\mu_{3}(i \hbar)^{3} B_{, p q r} U^{p j} U^{q k} U^{r l} A_{, j k l},
$$

and hence antisymmetry of our map:

$$
[A, B]=-[B, A]
$$

is achieved if and only if

$$
U^{j p}=-U^{p j}
$$

with $U^{j p}$ otherwise arbitrary for the time being. Moreover, bilinearity follows at once from the definition (2.1), i.e.

$$
[A, B+C]=[A, B]+[A, C]
$$

\section{GEOMETRY OF THE SPACE OF HISTORIES}

It is now helpful to summarize some key features of the geometry of the space $\Phi$ of histories. For this purpose, let us recall that $\Phi$ is endowed with a gauge-invariant and ultra-

local metric $\gamma[1]$. Gauge invariance means the vanishing of the Lie derivative of $\gamma$ along the vector fields $Q_{\alpha}$ that leave the action invariant as in Eq. (1.1), i.e.

$$
L_{Q_{\alpha}} \gamma=0
$$

while ultra-locality of $\gamma$ is expressed by

$$
\gamma_{i j^{\prime}}=\delta\left(x, x^{\prime}\right) f_{i j}
$$

with $f_{i j}$ independent of space-time derivatives of the fields $\varphi^{i}$. One can therefore build the operator

$$
\mathcal{F}_{\alpha \beta}=-Q_{\alpha}^{i} \gamma_{i j} Q_{\beta}^{j}
$$

whose Green functions satisfy

$$
\mathcal{F}_{\alpha \beta} G^{\beta \gamma}=-\delta_{\alpha}^{\gamma}
$$

It is then possible to introduce a family of connection $l$-forms on $\Phi$, i.e.

$$
\omega_{i}^{\alpha} \equiv \gamma_{i j} Q_{\beta}^{j} G^{\beta \alpha},
$$


which take values in the Lie algebra of the proper gauge group and play the role of 'inverses' of the $Q_{\alpha}^{i}$ with respect to field indices, in that

$$
Q_{\alpha}^{i} \omega_{i}^{\beta}=Q_{\alpha}^{i} \gamma_{i j} Q_{\gamma}^{j} G^{\gamma \beta}=-\mathcal{F}_{\alpha \gamma} G^{\gamma \beta}=\delta_{\alpha}^{\beta} .
$$

The fibre-adapted coordinates available can be denoted by $I^{A}$ and $K^{\alpha}$, where the $I$ 's label the fibres, i.e. the points in $\Phi / \mathcal{G}$, and are gauge-invariant, i.e. [1]

$$
Q_{\alpha} I^{A}=0,
$$

while the $K$ 's label the points within each fibre, and correspond to the choice of gauge-fixing functional in physical language. One usually singles out a base point $\varphi_{\star}$ in $\Phi$ and chooses the $K$ 's to be local functionals of the $\varphi$ 's of such a form that the 'matrix' [1]

$$
\widehat{F}_{\beta}^{\alpha}=Q_{\beta} K^{\alpha}=K_{, i}^{\alpha} Q_{\beta}^{i}
$$

is actually a non-singular differential operator at and in a neighbourhood of $\varphi_{\star}$. The operator (3.8) is the ghost operator [7, 8] of modern quantum field theory, and its Green functions are denoted by $\widehat{G}^{\beta \gamma}$. The latter solve the equation

$$
\widehat{F}_{\alpha \beta} \widehat{G}^{\beta \gamma}=-\delta_{\alpha}^{\gamma},
$$

and can obey one of the various boundary conditions defining hyperbolic Green functions (e.g. advanced, or retarded, or Feynman).

The invertible gauge-field operator $F_{i j}$ mentioned in Sec. 1 can be written in the form (hereafter we assume that the loop expansion of the 〈out|in $\rangle$ amplitudes is performed [1])

$$
F_{i j}=S_{, i j}+K_{, i}^{\alpha} \Omega_{\alpha \beta} K_{, j}^{\beta},
$$

where $\Omega_{\alpha \beta}$ is a non-singular $\varphi$-dependent local distribution having the gauge transformation law [2]

$$
\delta \Omega_{\alpha \beta}=\Omega_{\alpha \beta, i} Q_{\gamma}^{i} \delta \xi^{\gamma}=-\left(\Omega_{\delta \beta} C_{\gamma \alpha}^{\delta}+\Omega_{\alpha \delta} C_{\beta \gamma}^{\delta}\right) \delta \xi^{\gamma} .
$$

\section{THE NEW FORM OF $U^{j p}$}

We now try to build the antisymmetric $U^{j p}$ in (2.1) in such a way that it involves only the concepts defined so far, it yields a Poisson bracket when the space $\mathcal{F}(\Phi)$ of section 2 
is restricted to the space of gauge-invariant functionals satisfying Eq. (1.7) (this restriction being the one for which also the Peierls map becomes a Poisson bracket), and has welldefined gauge transformation properties (see Sec. 5). By virtue of these requirements, we are led to consider

$$
U^{j p} \equiv Q_{\alpha}^{[j} F_{l}^{p]} Q_{\beta}^{l} \widehat{G}^{\alpha \beta},
$$

while bearing in mind, from (2.1), also the Ward identities obtained by functional differentiation of (1.7), i.e., for $Z=A, B$,

$$
\begin{gathered}
Z_{, i j} Q_{\alpha}^{i}+Z_{, i} Q_{\alpha, j}^{i}=0 \\
Z_{, i j k} Q_{\alpha}^{i}+Z_{, i j} Q_{\alpha, k}^{i}+Z_{, i k} Q_{\alpha, j}^{i}+Z_{, i} Q_{\alpha, j k}^{i}=0
\end{gathered}
$$

where (4.2) and (4.3) are just two of the infinitely many Ward identities available. In light of (2.1) and (4.1), (4.3), we find

$$
\begin{aligned}
& A_{, j k l} U^{j p} U^{k s} U^{l r} B_{, p s r}=-\frac{1}{2}\left(A_{, j k} Q_{\alpha, l}^{j}+A_{, j l} Q_{\alpha, k}^{j}\right) F_{l}^{p} Q_{\beta}^{l} \widehat{G}^{\alpha \beta} B_{, p s r} U^{k s} U^{l r} \\
+ & \frac{1}{2} A_{, j k l} F_{l}^{j} Q_{\beta}^{l} \widehat{G}^{\alpha \beta}\left(B_{, p s} Q_{\alpha, r}^{p}+B_{, p r} Q_{\alpha, s}^{p}\right) U^{k s} U^{l r} .
\end{aligned}
$$

We have here exploited the linear dependence of $Q_{\alpha}^{i}$ on field variables for all type-I theories [1], i.e.

$$
Q_{\alpha, j k}^{i}=0
$$

Equation (4.4) can be reduced to an equation where only one functional derivative of $A$ and two functional derivatives of $B$ occur (or the other way around), by virtue of the Ward identities (4.2) and (4.3), but it already displays a very important property: our definition (4.1) can be used to obtain an 'addition' to the Peierls map. The term (4.4) does not vanish for generic type-I theories (e.g. Yang-Mills or general relativity), but it vanishes for Maxwell theory, where $Q_{\alpha}^{i}$ reduces to [1]

$$
Q_{\mu}\left(x, x^{\prime}\right)=-\delta_{, \mu}\left(x, x^{\prime}\right)
$$

which implies that the $Q_{\alpha}^{i}$ are independent of field variables for Maxwell theory, i.e. $Q_{\alpha, j}^{i}=0$ in this case. 


\section{GAUGE TRANSFORMATION LAW OF $U^{j p}$}

Under the infinitesimal gauge transformations (1.4), the generators $Q_{\alpha}^{i}$, gauge-field operator $F_{i j}$ and ghost Green function $\widehat{G}^{\alpha \beta}$ transform according to [1, 2]

$$
\begin{gathered}
\delta Q_{\alpha}^{i}=Q_{\alpha, r}^{i} Q_{\gamma}^{r} \delta \xi^{\gamma}, \\
\delta F_{i j}=-\left(F_{k j} Q_{\gamma, i}^{k}+F_{i k} Q_{\gamma, j}^{k}\right) \delta \xi^{\gamma}, \\
\delta \widehat{G}^{\alpha \beta}=\left(C_{\gamma \delta}^{\alpha} \widehat{G}^{\delta \beta}-\widehat{G}_{\delta}^{\alpha} C_{\gamma}^{\delta \beta}\right) \delta \xi^{\gamma} .
\end{gathered}
$$

Thus, the antisymmetric $U^{j p}$ defined in (4.1) has a well defined gauge transformation law. By virtue of (5.1)-(5.3), such a law can be eventually cast in the form

$$
\begin{aligned}
\delta U^{j p} & =\frac{1}{2}\left\{\left(Q_{\alpha, r}^{j} Q_{\gamma}^{r} F_{l}^{p}-Q_{\alpha, r}^{p} Q_{\gamma}^{r} F_{l}^{j}\right) Q_{\beta}^{l} \widehat{G}^{\alpha \beta}\right. \\
& +\left[-Q_{\alpha}^{j}\left(F_{k l} Q_{\gamma,}^{k}{ }^{p}+F_{k}^{p} Q_{\gamma, l}^{k}\right)+Q_{\alpha}^{p}\left(F_{k l} Q_{\gamma,}^{k}{ }^{j}+F_{k}^{j} Q_{\gamma, l}^{k}\right)\right] Q_{\beta}^{l} \widehat{G}^{\alpha \beta} \\
& +\left(Q_{\alpha}^{j} F_{l}^{p}-Q_{\alpha}^{p} F_{l}^{j}\right) Q_{\beta, r}^{l} Q_{\gamma}^{r} \widehat{G}^{\alpha \beta} \\
& \left.+\left(Q_{\alpha}^{j} F_{l}^{p}-Q_{\alpha}^{p} F_{l}^{j}\right) Q_{\beta}^{l}\left(C_{\gamma \delta}^{\alpha} \widehat{G}^{\delta \beta}-\widehat{G}_{\delta}^{\alpha} C_{\gamma}^{\delta} \beta\right)\right\} \delta \xi^{\gamma} .
\end{aligned}
$$

In the particular (but relevant) case of Maxwell theory, $U^{j p}$ is therefore gauge-invariant because $Q_{\alpha, j}^{i}=0$ as we said after (4.6), and the structure constants $C_{\beta \gamma}^{\alpha}$ vanish in the Abelian case.

Note also that the definition (2.1) can be generalized according to

$$
[A, B] \equiv i \hbar(A, B)+\mu_{1} i \hbar A_{, j} U^{j k} B_{, k}+\mu_{3}(i \hbar)^{3} A_{, j k l} U^{j p} U^{k q} U^{l r} B_{, p q r}+\mathrm{O}\left(\hbar^{5}\right),
$$

where, for $A$ and $B$ obeying (1.7), $A_{, j} U^{j k} B_{, k}$ vanishes for all type-I theories, by virtue of (4.1), whereas higher-order terms only vanish in the Abelian case. With our notation, $\mathrm{O}\left(\hbar^{5}\right)$ denotes a finite (or possibly infinite) number of contributions, of odd degree $\geq 5$ in $\hbar$.

As far as antisymmetric bilinear maps are concerned, the definition (5.5) might be further generalized along the lines suggested in Ref. [2], i.e. by including

$$
A \exp \left[\frac{i \hbar}{2} \frac{\overleftarrow{\delta}}{\delta \varphi^{j}} \widetilde{G}^{j k} \frac{\vec{\delta}}{\delta \varphi^{k}}\right] B-B \exp \left[\frac{i \hbar}{2} \frac{\overleftarrow{\delta}}{\delta \varphi^{j}} \widetilde{G}^{j k} \frac{\vec{\delta}}{\delta \varphi^{k}}\right] A
$$

The formal expansion of this map yields

$$
i \hbar(A, B)+\frac{(i \hbar / 2)^{3}}{3 !} V_{A B}+\mathrm{O}\left(\hbar^{5}\right)
$$


where, on defining

$$
W_{l}^{k}(P) \equiv\left(P_{, j} \widetilde{G}^{j k}\right)_{, l}, P=A, B
$$

one finds

$$
V_{A B}=-2 W_{l}^{k}(A)\left(\widetilde{G}_{, n}^{l m} W_{m}^{n}(B)\right)_{, k}-2 W_{l}^{k}(A)_{, n}\left(\widetilde{G}^{l m} W_{m}^{n}(B)\right)_{, k} .
$$

It is therefore clear that higher orders in $\hbar$ bring in infinitely many functional derivatives of the supercommutator $\widetilde{G}^{j k}$. This is certainly interesting in the investigation of the most general antisymmetric bilinear map, but not obviously useful if one wants to obtain eventually a Poisson bracket on gauge-invariant functionals (cf. the important work in Ref. [9]).

\section{CONCLUDING REMARKS AND OPEN PROBLEMS}

The Peierls bracket [6, 10, 11] has been applied in the modern literature on the manifestly covariant [12, 13, 14, 15, 16, 17, 18, 19, 20, 21] approach to quantization of gauge theories, including gravity, and some authors have even gone so far as to suggest that the Peierls bracket can be used to actually define the functional integral itself [20].

On the other hand, in ordinary quantum mechanics, the Poisson bracket can be obtained from the first-order (in $\hbar$ ) expansion of the Moyal bracket, and hence we have tried to understand whether the Peierls map for gauge theories can be suitably generalized. Contrary to our original expectations [2], the extension here proposed is not of the Moyal type, since we have not studied the phase-space formulation of type-I gauge theories, but rather their space-of-histories formulation.

Our findings are expressed by the definitions (2.1) and (4.1): the geometry of the space of histories for type-I gauge theories makes it possible to obtain an antisymmetric bilinear map that is richer than the Peierls map. At that stage, restriction to gauge-invariant functionals of the fields reduces (2.1) to the Peierls bracket only in the case of Maxwell theory. Our construction is richer than Peierls's if one just looks at antisymmetric bilinear maps, but is considerably weaker if one looks for Poisson brackets on the space of gauge-invariant field functionals. At least two outstanding problems are therefore in sight:

(i) How to improve the definition (2.1) so that it gives a Poisson bracket different from the Peierls bracket for all type-I gauge theories, when restricted to the space of gauge-invariant 
functionals. Should one instead look at maps having the general form (cf. Ref. [5] in ordinary quantum mechanics)

$$
[A, B](\varphi) \equiv \int L(\varphi, \chi, \psi ; \widetilde{G})(A(\chi) B(\psi)-B(\chi) A(\psi)) d \mu(\chi, \psi)
$$

where $d \mu(\chi, \psi)$ is a measure on the space of histories, and try to work out the form of the kernel $L(\varphi, \chi, \psi ; \widetilde{G})$ ?

(ii) Suppose one starts instead from a phase-space formulation of type-I gauge theories. Within this framework, the formal analogy with ordinary quantum mechanics on phase space might be exploited to find a suitable Moyal bracket, that should be proportional to the Peierls bracket to lowest order in $\hbar$. For this purpose, we plan to study first some examples borrowed from classical and quantum dynamics with just one pair of $(q, p)$ variables [22], to begin with. Can the resulting Moyal bracket be re-expressed in terms of position variables only? Does this shed new light on our goal of generalizing the Peierls bracket?

There is therefore room left for a lot of further work, and the hope remains that the

space-time approach to quantum field theory [13] might be extended so as to understand what is a deeper foundation of Peierls brackets [6] and gauge-invariant commutators [12].

\section{Acknowledgments}

We are indebted to G. Marmo for inspiring conversations, and to J. Gracia-Bondia and P. Vitale for correspondence. Our work has been partially supported by PRIN SINTESI.

[1] B.S. DeWitt The global approach to quantum field theory, International Series of Monographs on Physics 114 Clarendon Press, Oxford, 2003 (IMPHA,114,1); B.S. DeWitt in: 50 Years of Yang-Mills theory, ed. G. 't Hooft, World Scientific, Singapore, 2005.

[2] G. Esposito and C. Stornaiolo, hep-th/0607114.

[3] J.E. Moyal, Proc. Camb. Phil. Soc. 45 (1949) 99.

[4] T.F. Jordan and E.C.G. Sudarshan, Rev. Mod. Phys. 33 (1961) 515.

[5] J.M. Gracia-Bondia, F. Lizzi, G. Marmo and P. Vitale, JHEP 0204 (2002) 026.

[6] R.E. Peierls, Proc. R. Soc. Lond. A214 (1952) 143. 
[7] L.D. Faddeev and V.N. Popov, Phys. Lett. B25 (1967) 29.

[8] B.S. DeWitt, Phys. Rev. 162 (1967) 1195.

[9] A.C. Hirshfeld and P. Henselder, Am. J. Phys. 70, 537 (2002); Ann. Phys. (N.Y.) 298, 382 (2002).

[10] B.S. DeWitt, Dynamical theory of groups and fields, Gordon \& Breach, New York, 1965.

[11] G. Bimonte, G. Esposito, G. Marmo, C. Stornaiolo, Int. J. Mod. Phys. A18 (2003) 2033.

[12] B.S. DeWitt, Phys. Rev. Lett. 4 (1960) 317.

[13] B.S. DeWitt, The spacetime approach to quantum field theory, in Relativity, Groups and Topology II, eds B.S. DeWitt and R. Stora, 381-738, North-Holland, Amsterdam, 1984.

[14] J.E. Nelson and T. Regge, Ann. Phys. (N.Y.) 166 (1986) 234.

[15] C. Crnkovic and E. Witten, in: Three Hundred Years of Gravitation, eds. S.W. Hawking and W. Israel, Cambridge University Press, Cambridge, 1987.

[16] G. Barnich, M. Henneaux and C. Schomblond, Phys. Rev. D44 (1991) 939.

[17] D. Marolf, Ann. Phys. (N.Y.) 236 (1994) 374; 236 (1994) 392.

[18] I.V. Kanatchikov, Int. J. Theor. Phys. 40 (2001) 1121.

[19] M. Dütsch and K. Fredenhagen, Commun. Math. Phys. 243 (2003) 275.

[20] B.S. DeWitt and C. DeWitt-Morette, Ann. Phys. (N.Y.) 314 (2004) 448.

[21] H. Ozaki, Ann. Phys. (N.Y.) 319 (2005) 364.

[22] G. Esposito, G. Marmo and C. Stornaiolo, work in preparation. 